\title{
PROJETO, IMPLEMENTAÇÃO E CONTROLE DE UMA PLATAFORMA AEROSTÁTICA COM JATOS DE AR
}

\author{
WILIAN L. CAMPESATO ${ }^{1}$, VALDEMIR CARRARA ${ }^{2}$, GUILHERME A. SIQUELI $^{2}$, ANDRÉ FENILI $^{1}$ \\ 1. Centro de Engenharia, Modelagem, e Ciências Sociais Aplicadas, Universidade Federal do ABC \\ Av. dos Estados, 5001, Bairro Bangu, Santo André - SP - Brasil. CEP 09210-580 \\ E-mails: wilian.campesatolufabc.edu.br; andre.fenilieufabc.edu.br;
}

\section{Coordenação Geral de Engenharia e Tecnologia Espacial, Divisão de Mecânica Espacial e Controle, Instituto Nacional de Pesquisas Espaciais \\ Av. dos Astronautas, 1758, Jd da Granja, São Jose dos Campos - SP - Brasil. CEP 12225-010 \\ E-mails: valeinpe.dem.br; guilherme.siquelilinpe.br}

\begin{abstract}
This work aims to design, to implement and to control an air bearing platform by means of compressed air jets in order to simulate the attitude control of satellites using gas jet actuators. To simulate the space environment, the control system was implemented in an aerostatic bearing platform, which allows simulating an environment of low friction, similar to that found in the satellite orbits. This paper describes the design procedures, the requirements, the aerostatic platform, the control and communication scheme, the static balancing, the mathematical modeling, as well as discusses about simulations performed using bang-bang type control. This project aims to achieve an experimental environment in which various control laws based on gas jets and using various sensors can be tested, both to serve as a prototype to a real embedded system as to trial new control techniques besides academic use.
\end{abstract}

Keywords_ Aerostatic platform, attitude control, air bearing platform, artificial satellite.

\begin{abstract}
Resumo_Este trabalho tem por objetivo o projeto, implementação e controle de uma plataforma sustentada por mancal aerostático usando jatos de ar comprimido, a fim de simular o controle de atitude de satélites artificiais empregando atuadores de jatos de gás. Para simular o ambiente espacial, o sistema de controle foi implantado numa mesa (plataforma) de mancal aerostático, a qual permite simular um ambiente de baixo atrito, semelhante àquele encontrado nas órbitas de satélites. Neste trabalho são descritos os procedimentos de projeto, os requisitos, a plataforma aerostática, o esquema de controle e comunicação, o balanceamento estático, o modelamento matemático, bem como se discorre sobre simulações realizadas de controle tipo bang-bang. Este projeto visa conseguir um ambiente experimental no qual diversas leis de controle baseadas em jatos de gás e utilizando diversos sensores possam ser testadas, tanto para servir como protótipo a um sistema real embarcado em satélites quanto para experimentações de novas técnicas de controle, além de uso acadêmico.
\end{abstract}

Palavras-chave— Plataforma aerostática, controle de atitude, mesa de mancal a ar, satélite artificial.

\section{Introdução}

Segundo Arantes (2005) a orientação de um satélite em relação a um sistema de referência conhecido é denominada atitude e o movimento de rotação em torno do seu centro de massa é denominado movimento de atitude. De acordo com Hughes (1978) e Wertz (1986), a atitude e o movimento de atitude especificam a orientação espacial e o movimento rotacional em torno do centro de massa do satélite. Os subsistemas de controle de atitude de um veículo espacial são as partes deste veículo responsáveis pela sua orientação em relação a um sistema de coordenadas externo, alcançada por meio do movimento de atitude do veículo. Atualmente, satélites artificiais, sondas e naves espaciais possuem sistemas de controle de atitude (ACS) compostos basicamente por computadores de bordo utilizados como elementos controladores, atuadores para gerar os torques comandados pelo controlador e sensores para estimar a atitude e desvios na atitude. Normalmente os veículos espaciais, principalmente satélites artificiais, possuem mais de um tipo de atuador em seus sistemas. Durante uma missão espacial, a seleção entre a utilização de um ou de outro depende de fatores tais como velocidade de atuação, amplitude do ângulo necessário para a manobra de atitude, tempo necessário para a execução da manobra e gasto de energia. Sistemas compostos por jatos de gás são empregados para deslocamentos em grandes ângulos, normalmente em caráter emergencial, e possuem grande gasto de energia, que neste caso é restrita ao tanque de combustível. Sistemas compostos por rodas de reação e bobinas magnéticas, por outro lado, são empregados para deslocamentos de pequenos ângulos em maiores períodos de tempo, ou para ajustes precisos após a utilização de jatos de gás, e possuem pequeno gasto de energia, que é reposta pelos painéis fotovoltaicos. Este trabalho tem por objetivo o projeto, implementação e controle de uma plataforma sustentada por mancal aerostático de baixo atrito usando jatos de ar comprimido, a fim de simular o controle de atitude de satélites artificiais empregando atuadores de jatos de gás. Para simular o ambiente espacial, o sistema de controle foi implantado numa mesa (plataforma) de mancal aerostático com 3 graus de liberdade. Como principal objetivo almeja-se conseguir, ao fim do projeto, um ambiente experimental no qual diversas leis de controle baseadas em jatos de gás e utilizando diversos sensores possam ser testadas, tanto para servir como protótipo a um sistema real 
embarcado em satélites quanto para experimentações de novas técnicas de controle.

\section{Descrição do experimento}

Este trabalho foi desenvolvido no Laboratório de Simulações (LabSim) do INPE (Instituto Nacional de Pesquisas Espaciais) em São José dos Campos - SP. A plataforma ou mesa aerostática utilizada neste projeto foi projetada e construída no INPE (Cardieri e Varotto, 1987), para testar o amortecedor de nutação dos satélites SCD1 e SCD2 (Fonseca e Souza, 1989, 1990). Uma mesa de mancal aerostático, também chamada de plataforma de mancal a ar, ou ainda mesa ou plataforma de mancal esférico, é um dispositivo no qual uma calota esférica sólida geralmente de aço flutua sobre um colchão de ar comprimido, formado em uma cavidade esférica que se ajusta perfeitamente à calota, como mostrado esquematicamente na figura 1. A mesa permite simular um ambiente de baixo atrito, semelhante àquele encontrado nas órbitas de satélites em torno da Terra. Ela permite que a plataforma gire livremente em torno do eixo vertical, mas o movimento em torno dos eixos horizontais é limitado pela necessidade da base de suporte.

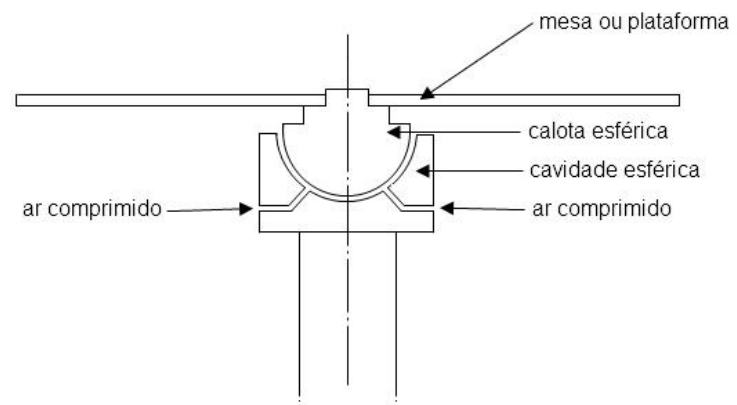

Figura 1. Esquema em corte de um mancal esférico.

Foram fixadas na plataforma oito válvulas pneumáticas direcionais, uma válvula reguladora de pressão, dois reservatórios de ar comprimido, um manômetro, um engate rápido para recarga dos reservatórios, uma bateria de $12 \mathrm{~V}$, uma eletrônica de controle (PC104) e potência, um transmissor-receptor wireless, uma unidade inercial (AHRS - Attitude and Heading Reference System) composta por três giroscópios de estado sólido, três acelerômetros e um magnetômetro triaxial, cabeamento elétrico e tubulações pneumáticas, além de suportes para alguns componentes e finalmente massas de balanceamento. A foto da figura 2 ilustra a plataforma integrada. Todos os componentes foram fixados de maneira a fazer com que o centro de massa da plataforma fique o mais próximo possível do centro geométrico da meia-esfera do mancal, reduzindo assim torques residuais devido ao desbalanceamento. Foram realizados estudos mecânicos para a estimação da inércia e balanceamento estático da mesa; estudos pneumáticos para a estimação de forças e torques gerados pelos jatos de ar; e simulação computacional do controle da atitude da plataforma utilizando o programa "PROPAT - Satellite Attitude and Orbit Toolbox for Matlab" (Carrara, 2012).

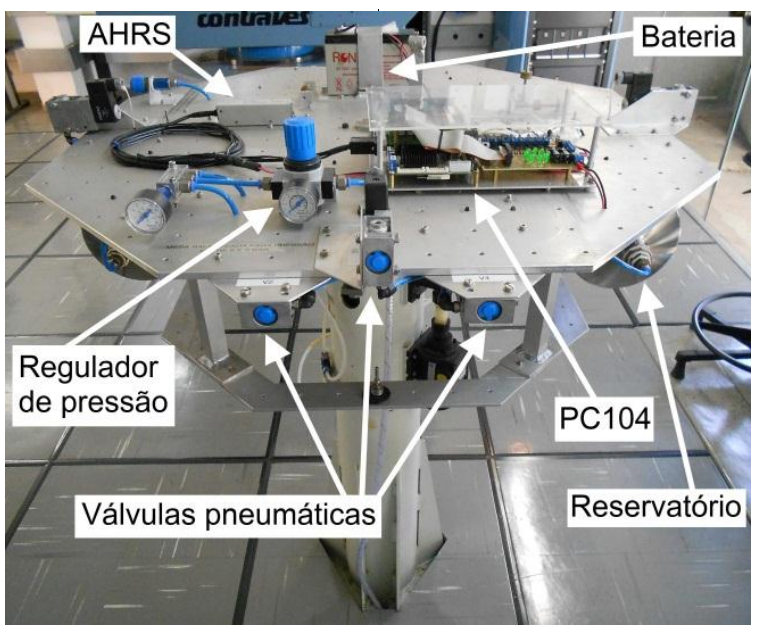

Figura 2. Mesa de mancal aerostático.

Os principais requisitos atendidos por este projeto são:

1) Simular o ambiente espacial numa mesa de mancal aerostático, com torques residuais compatíveis com aqueles gerados pelos jatos de ar;

2) Gerar torques em três eixos ortogonais;

3) Controlar a atitude da plataforma por meio de de jatos de ar comprimido liberados por válvulas pneumáticas, acionadas pela eletrônica de controle;

4) Disponibilizar um ambiente de processamento digital embarcado na plataforma e autônomo;

5) Permitir a visualização e monitoração da plataforma em qualquer instante por meio de telemetria wireless. Cablagem e linhas de transmissão são utilizadas apenas para configuração e cargas da plataforma (ar comprimido, baterias, programas de controle) quando esta não estiver em operação;

6) Garantir um alinhamento dos momentos principais de inércia com os eixos geométricos da plataforma por meio de balanceamento dinâmico;

7) Produzir algoritmos para determinação autônoma de atitude da plataforma.

\section{Desenvolvimento do projeto}

\subsection{Eletrônica de controle}

Como eletrônica de controle utilizou-se um PC104. O controlador comunica-se por meio de um transmissor wireless com um microcomputador externo. A plataforma conta com uma interface de potência entre o controlador e as válvulas solenoides, uma unidade inercial AHRS (Attitude and Heading Reference System) da Innalabs (Innalabs, 2009). A figura 3 ilustra o esquema das interfaces entre os equipamentos. Durante o experimento, o computador em- 
barcado envia para o computador externo a telemetria de posicionamento e velocidades recebida dos sensores.

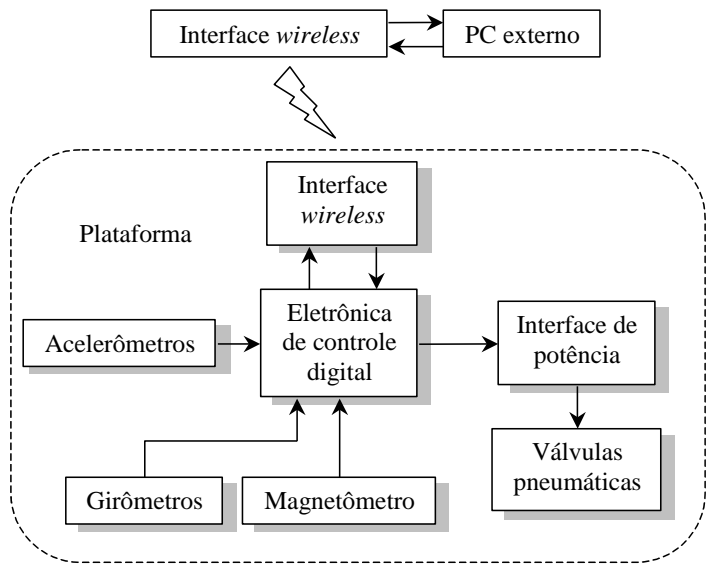

Figura 3. Esquema de conexões eletrônicas do experimento.

\subsection{Definição dos eixos e posicionamento das válvu- las pneumáticas}

As válvulas pneumáticas agem como atuadores do experimento, fornecendo torques para a plataforma. O esquema do posicionamento destas, da direção dos jatos de ar, e do sistema de coordenadas da plataforma é apresentado na figura 4 . Foram fixadas 8 válvulas na plataforma. Cada par gera torque em direções opostas nos eixos $x$ e $y$. No eixo $\mathrm{z}$ foram fixados dois pares, sendo que cada par trabalha em conjunto e gera torque em um único sentido.

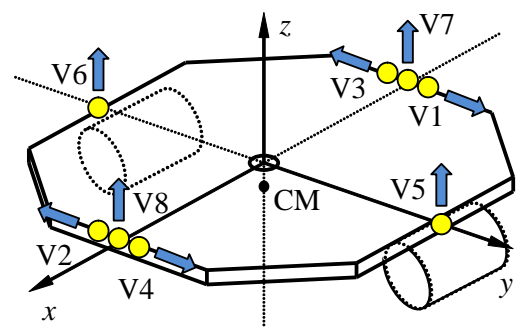

Figura 4. Esquema do posicionamento das válvulas, do centro de massa CM aproximado (centro da meia-esfera do mancal), e a direção dos jatos de ar.

\subsection{Massa de ar nos reservatórios}

Para estimar a massa de ar comprimido nos reservatórios, parte-se da lei dos gases perfeitos que fornece a relação:

$$
p V=\frac{m}{M} R T
$$

sendo $V=101$ o volume, $m$ a massa, $R$ é a constante dos gases $\left(R=8.314472 \mathrm{~m}^{3} \mathrm{~Pa} / \mathrm{K} \mathrm{mol}\right), M=28,98$ $\mathrm{g} / \mathrm{mol}$ é a massa molar do ar, $T=25^{\circ} \mathrm{C}=298^{\circ} \mathrm{K}$ é a temperatura absoluta, e $p$ a pressão (absoluta), na qual a pressão mínima de operação $p_{\min }=2 \mathrm{bar}=2$ $10^{5} \mathrm{~Pa}$ e pressão máxima de operação $p_{\max }=12 \mathrm{bar}=$
$1210^{5} \mathrm{~Pa}$. A massa de ar nos reservatórios resulta em $m_{\min }=0,0234 \mathrm{~kg}$ e $m_{\max }=0,1403 \mathrm{~kg}$. A massa de ar a ser considerada no cálculo deve ser igual à diferença entre a massa de ar no reservatório e a massa de ar deslocada (empuxo), que vale $0,0117 \mathrm{~kg}$. Logo, temse $\Delta m_{\min }=0,0117 \mathrm{~kg}$ e $\Delta m_{\max }=0,1287 \mathrm{~kg}$, sendo que cada reservatório armazena metade desta massa. Uma estimativa do deslocamento do centro de massa da plataforma quando o reservatório é esvaziado (da pressão máxima para a pressão mínima) forneceu um valor de $0,2 \mathrm{~mm}$ na direção $z$. Embora este deslocamento provoque um desbalanceamento na plataforma, considera-se que a mudança seja aceitável por ser pequena.

\subsection{Força e torque nas válvulas}

Segundo o catálogo das válvulas pneumáticas, a vazão nominal da válvula é de 500 1/min com pressão de trabalho de 1,5 a 8 bar. Supondo uma pressão de operação relativa de 2 bar, com um consumo de 500 1/min tem-se que o diâmetro da abertura original da válvula deve ser um pouco maior do que $4 \mathrm{~mm}$. Em satélites de até $500 \mathrm{~kg}$, o torque gerado por propulsores em geral fica compreendido entre $0,1 \mathrm{Nm}$ a 1 $\mathrm{Nm}$. Para gerar um torque reduzido e também minimizar o consumo fixou-se tampões com orifícios de $0,8 \mathrm{~mm}$ na saída das válvulas. Estima-se que, com esta abertura, a força resultante será de $F=0,1 \mathrm{~N}$, e o torque será de $T_{q}=0,0445 \mathrm{Nm}$, cuja magnitude é compatível com a inércia da plataforma, além de prover um menor consumo de ar.

\subsection{Balanceamento estático}

Estando todos os componentes fixados na mesa, o objetivo do processo de balanceamento estático é levar o centro de massa (consequentemente também o centro de gravidade) de todo o conjunto o mais próximo possível do centro geométrico da meia esfera do mancal, a fim de que o sistema não apresente torques ou movimentos relativos aparentes, isto é, não apresente movimentos característicos de pêndulo simples (equilíbrio estável) ou pêndulo invertido (equilíbrio instável). Isto garante que a plataforma irá comportar-se de maneira similar a um satélite em órbita da Terra. Cabe ressaltar que não há como evitar a influência das partes móveis das válvulas, bem como a migração do centro de massa da mesa quando os reservatórios de ar forem gradativamente esvaziados, tendo-se assim que conviver com estes efeitos. As massas de balanceamento foram fixadas em quatro pontos simétricos do suporte das massas de balanceamento, nos planos $x-z$ e $y$ - $z$, de tal forma a alinhá-las com os eixos coordenados. Para efetuar o balanceamento, numa primeira etapa, foram adicionadas massas à mesa de forma a levar o centro de massa para baixo do centro geométrico do mancal e ao mesmo tempo alinhá-lo com o eixo $z$ a fim de que a plataforma passasse a se comportar como um pêndulo simples. Feito isso, a segunda etapa consistiu na retirada gradual de massa simetricamente aos quatro locais de fixação das massas de balanceamento para 
elevar o CG até este coincidir com o centro da meia esfera do mancal aerostático. Esta situação é detectada ao perceber-se que a mesa não mais se comporta de forma pendular. Alcançando-se aproximadamente este ponto, o sistema passa a apresentar torque de desbalanceamento extremamente baixo e, então, os torques de atrito da mesa com o ar atmosférico e torques de atrito residual no mancal a ar ganham amplitude significativa. Não há como reduzir estes torques; entretanto, há como mensurá-los. Para baixas velocidades, torques de atrito da mesa com o ar atmosférico podem ser considerados lineares, cuja constante de proporcionalidade deve ser obtida experimentalmente em trabalhos futuros. $\mathrm{O}$ torque de atrito residual é extremamente difícil de ser mensurado ou mesmo modelado, além de depender da orientação da mesa em relação ao mancal. Este torque possui um caráter aparentemente errático em virtude de turbilhonamentos que ocorrem quando o ar pressurizado flui pelo mancal. Entretanto, como sua amplitude é relativamente baixa, pode-se conviver com ele na execução de experimento durante os procedimentos de implementação e desenvolvimento da mesa. Devido aos movimentos permitidos pela mesa, pode-se afirmar que o balanceamento nas direções do plano da mesa ( $x$ e $y$ ) pode ser realizado facilmente, mas é bastante difícil distinguir se o centro de massa encontra-se acima ou abaixo do centro da esfera (eixo $z$ ). Para evidenciar este desalinhamento a mesa foi posta a girar manualmente sobre seu eixo $z$, mas de forma a que este eixo ficasse com um certo ângulo com relação à vertical. Sob tal condição, caso haja algum desbalanceamento residual neste eixo, a mesa deve apresentar um movimento de precessão similar ao de um pião inclinado. Se o balanceamento for perfeito não se observa tal movimento. $\mathrm{O}$ movimento de precessão pode ser facilmente observado já que a mesa leva dezenas de minutos antes que a velocidade angular caia em demasia. Além disso, o sentido de precessão, se horário ou anti-horário, fornece a indicação se o centro de massa encontra-se acima ou abaixo do centro da esfera.

\section{Controle, otimização e simulação}

\subsection{Modelagem e lei de controle}

Com a intenção de validar um esquema de controle por meio de simulação, decidiu-se implementar um controle bang-bang de posição-e-velocidade sugerido por Headrick (Wertz, 1978), que assume que o sinal para o atuador deve seguir um controle PD:

$$
u_{i}=\operatorname{sgn}\left(-k_{p} \theta_{i}+k_{p} \theta_{r i}-k_{d} \omega_{i}\right)
$$

onde $u_{i}$ é o sinal discreto $(+1,-1$ ou 0$)$ do torque a ser gerado no eixo $i$ (x,y ou z), $k_{p}$ e $k_{d}$ são os ganhos proporcional e derivativo do controlador, $\theta_{i}$ são os ângulos de Euler da atitude de uma transformação 123, $\theta_{r i}$ são os ângulos de referência, e $\omega_{i}$ são as componentes do vetor velocidade angular da mesa.
Contudo, este controle pode apresentar problemas de convergência sempre que $\theta_{i}$ passar por transições entre $-\pi$ e $\pi$ ou 0 e $2 \pi$, pois $u_{i}$ muda repentinamente sem necessariamente se anular. Para evitar isto, efetua-se primeiramente um controle para reduzir a velocidade angular quando ela for superior a uma velocidade angular limite $\omega_{\text {lim }}$, definida de forma a garantir que o controle de posição possa ser atingido em menos do que meia volta da mesa. Também, para evitar que as válvulas fiquem comutando rapidamente quando a atitude final for atingida, estabeleceu-se uma zona morta $k_{d b}$ (dead band) ao redor da referência de atitude:

$$
\begin{gathered}
\omega_{\text {lim }}=\sqrt{\frac{2 \pi T_{q}}{I_{\text {max }}}} \\
z_{i}=\left\{\begin{array}{l}
1, \text { se }\left|\theta_{i}\right|>k_{d b} \\
0, \text { caso contrário }
\end{array}\right.
\end{gathered}
$$

tal que $T_{q}$ é o torque gerado pelos atuadores, e $I_{\max }$ é o maior momento de inércia da mesa. A lei de controle fica então:

$u_{i}=\left\{\begin{array}{l}z_{i} \operatorname{sgn}\left(-k_{d} \omega_{i}\right), \text { se } \omega_{i}>\omega_{\text {lim }} \\ z_{i} \operatorname{sgn}\left(-k_{p} \theta_{i}+k_{p} \theta_{r i}-k_{d} \omega_{i}\right), \text { se } \omega_{i} \leq \omega_{\text {lim }}\end{array}\right.$

A dinâmica foi simulada por meio de integração numérica das equações de Euler para o movimento rotacional de corpos rígidos usando o pacote $P R O$ PAT (Carrara, 2012). A equação da dinâmica da atitude é dada por:

$$
\dot{\boldsymbol{\omega}}^{b}=\mathbf{I}^{-1}\left(\mathbf{T}_{q}-\boldsymbol{\omega}^{\times} \mathbf{I} \boldsymbol{\omega}\right)
$$

onde I é a matriz de inércia, $\mathbf{T}_{\mathrm{q}}=\mathrm{T}_{\mathrm{q}}\left(\mathrm{u}_{1}, \mathrm{u}_{2}, \mathrm{u}_{3}\right)^{\mathrm{T}}$ é o vetor de torques de controle (torques perturbadores desprezados), $\dot{\boldsymbol{\omega}}^{\boldsymbol{b}}$ é a aceleração angular (sistema de coordenadas fixado na mesa), e $\boldsymbol{\omega}^{x}$ é a matriz antisimétrica do produto vetorial da velocidade angular $\boldsymbol{\omega}$, definida por:

$$
\boldsymbol{\omega}^{\times}=\left(\begin{array}{ccc}
0 & -\omega_{3} & \omega_{2} \\
\omega_{3} & 0 & -\omega_{1} \\
-\omega_{2} & \omega_{1} & 0
\end{array}\right)
$$

A atitude foi integrada numericamente usando o integrador ODE45 que compõe a biblioteca de integradores do MATLAB, com erro relativo e erro absoluto ajustados para $10^{-8}$. A atitude foi integrada em quatérnios, cuja equação diferencial é:

$$
\dot{\mathbf{Q}}=\frac{1}{2} \mathbf{\Omega} \mathbf{Q}
$$

onde $\mathbf{Q}=(\boldsymbol{\varepsilon} \eta)^{T}$ é o quatérnio de atitude ( $\boldsymbol{\varepsilon}$ é a parte vetorial e $\eta$ é a parte escalar), e $\Omega$ é a matriz antissimétrica definida por: 


$$
\boldsymbol{\Omega}=\left(\begin{array}{cc}
-\boldsymbol{\omega}^{\times} & \boldsymbol{\omega} \\
-\boldsymbol{\omega}^{T} & 0
\end{array}\right)=\left(\begin{array}{cccc}
0 & \omega_{3} & -\omega_{2} & \omega_{1} \\
-\omega_{3} & 0 & \omega_{1} & \omega_{2} \\
\omega_{2} & -\omega_{1} & 0 & \omega_{3} \\
-\omega_{1} & -\omega_{2} & -\omega_{3} & 0
\end{array}\right)
$$

A conversão de quatérnios para ângulos de Euler de uma rotação 1-2-3 é realizada inicialmente convertendo quatérnios na matriz de rotação da atitude por meio de:

$$
\mathbf{C}=\left(\begin{array}{ccc}
\eta^{2}+\varepsilon_{1}^{2}-\varepsilon_{2}^{2}-\varepsilon_{3}^{2} & 2\left(\varepsilon_{1} \varepsilon_{2}+\eta \varepsilon_{3}\right) & 2\left(\varepsilon_{1} \varepsilon_{3}-\eta \varepsilon_{2}\right) \\
2\left(\varepsilon_{1} \varepsilon_{2}-\eta \varepsilon_{3}\right) & \eta^{2}-\varepsilon_{1}^{2}+\varepsilon_{2}^{2}-\varepsilon_{3}^{2} & 2\left(\varepsilon_{2} \varepsilon_{3}+\eta \varepsilon_{1}\right) \\
2\left(\varepsilon_{1} \varepsilon_{3}+\eta \varepsilon_{2}\right) & 2\left(\varepsilon_{2} \varepsilon_{3}-\eta \varepsilon_{1}\right) & \eta^{2}-\varepsilon_{1}^{2}-\varepsilon_{2}^{2}+\varepsilon_{3}^{2}
\end{array}\right),
$$

e, a seguir, calculam-se os ângulos de Euler com as relações:

$$
\begin{gathered}
\theta_{1}=\arctan \left(\frac{-c_{32}}{c_{33}}\right), \\
\theta_{2}=\operatorname{arcsen}\left(c_{31}\right), \\
\theta_{3}=\arctan \left(\frac{-c_{21}}{c_{11}}\right),
\end{gathered}
$$

nas quais $c_{i j}$ representa o elemento da linha $i$ e coluna $j$ da matriz de atitude. A matriz de inércia da mesa foi calculada no MATLAB, usando para isso tanto medições diretas da massa dos diversos equipamentos integrantes da mesa quanto cálculos teóricos baseados na geometria destes equipamentos. O MATLAB permitiu também que fossem calculadas massas para balanceamento estático e dinâmico da mesa. Chegouse, com isso, à matriz de inércia dada por:

$\mathbf{I}=\left(\begin{array}{ccc}I_{1} & 0 & 0 \\ 0 & I_{2} & 0 \\ 0 & 0 & I_{3}\end{array}\right)=\left(\begin{array}{ccc}2,21 & 0 & 0 \\ 0 & 1,91 & 0 \\ 0 & 0 & 2,17\end{array}\right) \mathrm{kg} \mathrm{m}^{2}$

de onde se conclui que $I_{\max }=2,21 \mathrm{~kg} \mathrm{~m}^{2}$. O consumo de ar é proporcional ao tempo de funcionamento dos atuadores. Portanto, a condição de otimização consiste em minimizar a função:

$$
c=\sqrt{\sum_{t}[\mathbf{u}(t) \Delta t]^{2}}
$$

onde $\mathbf{u}(t)=\left(\begin{array}{lll}u_{1} & u_{2} & u_{3}\end{array}\right)^{T}$ e $\Delta t$ é o intervalo de discretização do controle. Nota-se que a integral do consumo foi substituída por uma somatória, já que o acionamento dos atuadores é discreto e o tempo de discretização é constante.

\subsection{Simulação}

Para simular o efeito das medidas do sensor de atitude, introduziu-se um ruído gaussiano nos ângulos de Euler compatível com aquele apresentado pelo sen- sor AHRS. Admitiu-se que este ruído possua média nula e desvio padrão de $1^{\circ}$ nas medidas angulares e de $10^{-3} \mathrm{rad} / \mathrm{s}$ na velocidade angular. Este ruído pode provocar a comutação aleatória do atuador quando este estiver próximo da referência, e, em virtude disso, adotou-se um valor para a zona morta de $2^{\circ}$, superior, portanto, ao desvio padrão do sensor. Escolheu-se atitude inicial baseada no experimento descrito na próxima seção, cuja atitude inicial foi $\left(\theta_{1}, \theta_{2}, \theta_{3}\right)=\left(2^{\circ}, 2^{\circ},-105^{\circ}\right)$, e $\left(\omega_{1}, \omega_{2}, \omega_{3}\right)=(0,0,0)$ $\mathrm{rad} / \mathrm{s}$, propagando-a por $200 \mathrm{~s}$ com passo de $1 / 10 \mathrm{~s}$. O principal objetivo foi obter um conjunto de ganhos para o controlador que minimizasse o consumo de ar comprimido numa manobra de aquisição e estabilização da atitude. Assim, considerando-se nulo o ruído gaussiano do sensor, chegou-se de forma empírica aos valores $k_{p}=0,2$ e $k_{d}=1,2$, com intervalo de discretização do controle em 0,1 s (frequência máxima de acionamento das válvulas de $10 \mathrm{~Hz}$ ). Para estes valores, foram obtidos os resultados apresentados nas figuras 5 e 6 , onde as cores vermelho, verde e azul nos gráficos representam, respectivamente, os eixos $x, y$ e $z$.
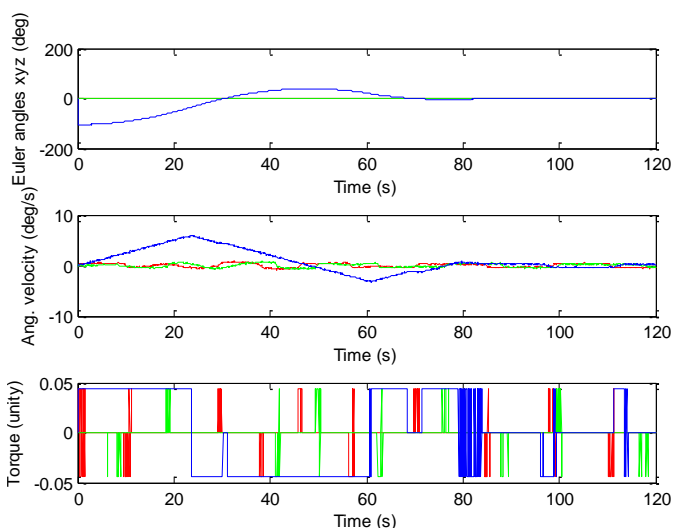

Figura 5. Ângulos de Euler, velocidade angular e torque das válvulas em função do tempo com consumo mínimo.

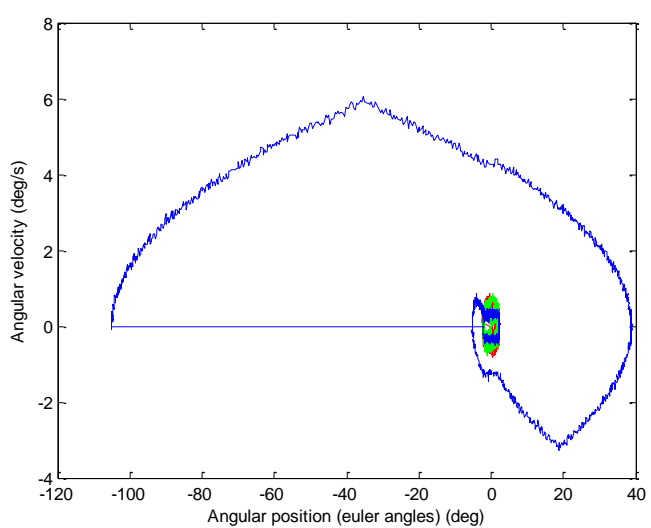

Figura 6. Plano de fase com ganhos que geram consumo mínimo.

Efetuou-se também simulação alterando-se o intervalo de discretização do controlador, que passou de 10 $\mathrm{Hz}$ para $1 \mathrm{~Hz}$ (tempo mínimo de acionamento das válvulas de 1 segundo). Observou-se que o consumo de ar nesta simulação foi bastante superior ao consumo apresentado quando as válvulas operavam em 
frequência de $10 \mathrm{~Hz}$. Esta frequência é limitada pela velocidade máxima de comutação das válvulas à pressão de 2 bar (indisponível no catálogo do fabricante). Sendo assim, considerou-se razoável utilizar a frequência trabalho como sendo de $10 \mathrm{~Hz}$.

\subsection{Teste real na mesa de mancal a ar}

Implementou-se na mesa um controlador PD descrito na Eq. (5) com $k_{p}$ e $k_{d}$ obtidos anteriormente na simulação. Os resultados são mostrados nas figuras 7 e 8 . A atitude de referência (ângulos de Euler nulos) foi atingida após cerca de 50 segundos a contar do início do experimento, como pode ser visualizado na figura 7, gráfico superior. A figura 7 inferior permite concluir que o torque gerado pelas válvulas é praticamente constante. $\mathrm{O}$ plano de fase da figura 8 permite identificar a trajetória efetuada pela plataforma sob a ação dos jatos até a estabilização na atitude de referência.
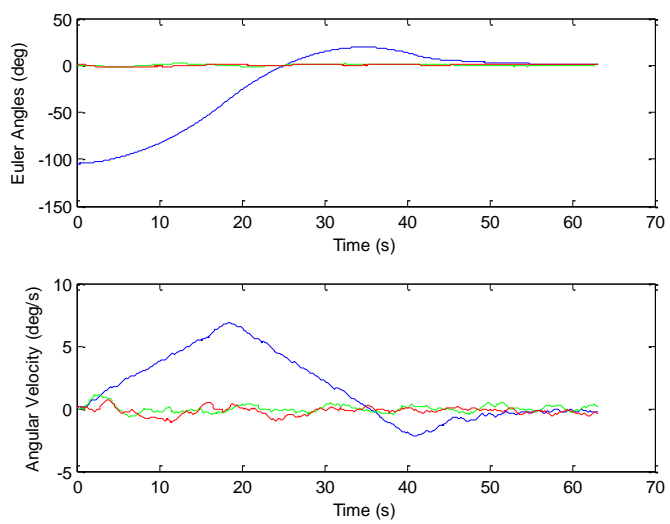

Figura 7. Dados reais de ângulos de Euler e velocidades angulares em função do tempo.

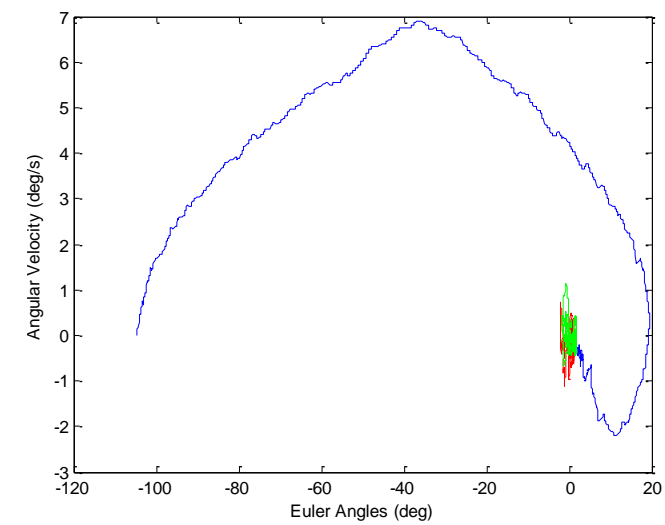

Figura 8. Plano de fase com dados reais.

\section{Conclusão}

Conclui-se que é viável simular o controle de atitude de satélites por meio de jatos de ar comprimido em ambiente laboratorial de baixo atrito, bem como para experimentações de novas técnicas de controle e uso acadêmico diverso. Alcançou-se balanceamento estático considerado aceitável para a simulação de controle de atitude em ambiente laboratorial. Os torques de atrito e de desbalanceamento são passíveis de serem contornados nas simulações, bastando incluí-los nos modelos dinâmicos a serem gerados em trabalhos futuros. É possível otimizar o gasto de ar comprimido (combustível) realizando-se ajustes dos ganhos do controlador, a fim de se obter menor consumo de ar para controlar a atitude da plataforma. Esse consumo também é bastante influenciado pela frequência de acionamento das válvulas, na qual observou-se que quanto maior a frequência (limitado pela velocidade máxima de comutação das válvulas), menor o consumo de ar.

\section{Agradecimentos}

Agradeço ao INPE, ao CNPq, ao Professor Valdemir Carrara pela oportunidade e paciência, ao aluno do Mestrado em Engenharia e Tecnologia Espacial Alexandre Macedo de Oliveira pelo grande auxílio no desenvolvimento do projeto, a meus pais pelo apoio e incentivo, e à UFABC pela oportunidade de estudar na "Universidade de ponta para o século XXI".

\section{Referências Bibliográficas}

ARANTES JR, G. Estudo comparativo de técnicas de controle de atitude em três eixos para satélites artificiais. 2005. 201 p. (INPE-12970TDI/1018). Dissertação (Mestrado em Engenharia e Tecnologia Espacial / Mecânica Espacial e Controle) - Instituto Nacional de Pesquisas Espaciais (INPE), São José dos Campos, 2005.

CARDIERI, L.V. C.; VAROTTO, S. E. C. Projeto de um simulador dinâmico com mancal esférico aerostático para testes de sistemas de controle de atitude de satélites artificiais. São José dos Campos: INPE, 1987, 5p. (INPE-4284-RE/1155).

CARRARA, V. PROPAT - Satellite Attitude and Orbit Toolbox for Matlab. 2012. Disponível em <http://www2.dem.inpe.br/val/projetos/propat/def ault.htm>. Acessado em 23 jun. 2013.

FONSECA, I.M.; SOUZA, P.N. Movimento rotacional de um corpo rígido dotado de um amortecedor de nutação anular viscoso - modelagem, simulação digital e validação experimental em um simulador dinâmico com mancal aerostático. In: DINAME, 1988; Congresso Brasileiro de Ciências Mecânicas, 1.989. Rio de Janeiro. p. 597-600.

FONSECA, I.M.; SOUZA, P.N. Partially filled viscous ring nutation damper dynamics analysis and experimental approach for modeling validation. International Symposium on Space Technology and Science, 17., 1990, Tokyo, Japan.

HUGHES, P.C. Spacecraft attitude dynamics. New York: John Wiley \& Sons, 1986. 564 p.

WERTZ, J. R. Spacecraft attitude determination and control. London, England: D. Reideil Publishing Company, 1978. 861 p. DOI: 10.1007/978-94-009-9907-7 